http://jmscr.igmpublication.org/home/ ISSN (e)-2347-176x ISSN (p) 2455-0450

crossref DOI: https://dx.doi.org/10.18535/jmscr/v7i11.36

Journal Of Medical Science And Clinical Research

\title{
Evaluation of infrastructure, implementation and bottlenecks in the Mission Indradhanush program in selected districts of Indore division (Madhya Pradesh)- A Cross sectional study
}

\author{
Authors \\ Dr Anand Kumar Patidar ${ }^{1}$, Dr Jageshwar Dayal Kori ${ }^{1}$, Dr Bhagwan Waskel ${ }^{2}$, \\ Dr A.K. Khatri ${ }^{2}$, Dr Sanjay Dixit ${ }^{3}$ \\ ${ }^{1}$ PG Resident, ${ }^{2}$ Associate Professor MGM Medical College Indore, ${ }^{3}$ Dean GMC Ratlam \\ *Corresponding Author \\ Dr Jageshwar Dayal Kori
}

\begin{abstract}
Background: Govt. of India, launched Mission Indradhanush in December 2014 to achieve more than 90\% full immunization coverage in the country. It focus on the areas with sub-centers where no auxiliary nurse midwife posted for more than three months, villages/areas with three or more consecutive missed routine immunization sessions, high risk areas. There were two main components of mission (1) Operational planning: By using Fixed and outreach and Mobile sessions (2) Communication planning.

Methods: It was a cross sectional study done (2016-17) in 3 randomly selected districts of Indore division where the Mission Indradhanush campaign was held. In each selected District 4 Planning unit (CHC/PHC, Total=12) \& 2 session site from each planning units (Total=24) were randomly selected. Data collection was done by using checklist based questionnaire.

Results: Most of the posts of health care providers involved in program was filled except 1 post of cold chain technician. Most of the health care providers were trained for their work. About at $86 \%$ sessions sites vaccines was delivered by alternate vaccine delivery system. Immunization card and updated due list by vaccinators was available at most of the session sites.

Conclusion: Basic infrastructure and logistics required for cold chain maintenance was available at most of the cold chain points. Material for vaccination like MCP cards, counter foils and updated due list were available at most of the session sites.
\end{abstract}

Keywords: Mission Indradhanush, ANM, ASHA, IPC, AVDs.

\section{Introduction}

Ministry of Health \& Family Welfare, Govt. of India, launched Mission Indradhanush in December 2014 to achieve more than $90 \%$ full immunization coverage in the country for closing immunity gaps and strengthen immunization coverage. It targets all children under the age of two years and pregnant women with all available vaccines. ${ }^{1}$ Over 27 million children, who live mainly in disadvantaged rural communities, are not reached by routine immunization services and significant variations in coverage exist between and within regions and countries. India records 5 lakh child deaths annually due to vaccine 
preventable diseases. Despite high childhood mortality rates due to vaccine preventable diseases, 30 percent of Indian children miss the benefits of full immunization every year. ${ }^{2}$

It focus on the areas with sub-centers where no auxiliary nurse midwife (ANM) posted for more than three months, villages/areas with three or more consecutive missed routine immunization sessions, high risk areas includes urban slums with migration, nomadic sites, brick kilns, construction sites, other migrant settlements (fisherman villages, riverine areas with shifting populations) underserved and hard to reach populations (forested and tribal populations, hilly areas etc.).

There were two main components of mission (1)Operational planning: By using Fixed and outreach sessions at health posts, primary health centers and district hospital and Mobile sessions where routine immunization coverage is weak and the small number of beneficiaries does not warrant an independent session to reach out to the unreached or poorly reached beneficiaries. (2) Communication Planning: For reaching out to communities and hard-to-reach populations and building trust in health care services. This calls for identifying communication methods or channels by IPC skill training, mass medias, IEC material, community and mother meetings and home visits by frontline health workers. ${ }^{3}$

\section{Aims \& Objectives}

1) To study infrastructure and implementation of Mission Indradhanush program.
2) To study the bottlenecks in implementation of Mission Indradhanush

\section{Methodology}

It was a cross sectional study done in 12 months of study period (March 2016-April 2017) in 3 randomly selected districts of Indore division where the Mission Indradhanush campaign was held. In each selected District 4 Planning unit $(\mathrm{CHC} / \mathrm{PHC}$, Total=12) \& 2 session site from each planning units (Total=24) were randomly selected. Data collection was done by using checklist based questionnaire which assess the infrastructure, implementation and bottlenecks of Mission Indradhanush at each cold chain points and at vaccination sites and by In-depth Interview of District Immunization Officer (DIO) at district level and Medical Officer (MO) at planning units. Data were collected and entered in Microsoft Excel sheet and analysed and results of the study is expressed in numbers and percentages in tabular form. Health Care Personnel of selected districts, planning units and session sites where Mission Indradhanush program was conducted and who gave consent were included in study and districts not selected for Mission Indradhanush and health personnel not gave consent were excluded for this study

\section{Results}

Present study was done to assess the infrastructure, implementation and bottlenecks of Mission Indradhanush at randomly selected 3 districts, 12 Planning units and 24 session sites.

Table No.1: Information about health care providers involved in Mission Indradhanush in 3 selected districts

\begin{tabular}{|l|l|c|c|c|}
\hline S.NO. & Health resources & Total & Post filled & Post vacant \\
\hline $\mathbf{1}$ & District Immunization Officer & 3 & 3 & 0 \\
\hline $\mathbf{2}$ & Medical Officer & 12 & 12 & 0 \\
\hline $\mathbf{3}$ & Cold Chain Handlers & 15 & 15 & 0 \\
\hline $\mathbf{4}$ & Cold Chain Technician & 3 & 2 & 01 \\
\hline $\mathbf{5}$ & ANM & 1195 & 1195 & 0 \\
\hline $\mathbf{6}$ & ASHA & 3494 & 3494 & 0 \\
\hline
\end{tabular}

Above table depicts that most of the posts of health care providers involved in mission indradhanush program was filled except 1 post of cold chain technician was vacant. 
Table No.2: Information about training status of health care provider involved in Mission Indradhanush

\begin{tabular}{|l|l|c|c|c|}
\hline At District level & Total & Trained & Not Trained \\
\hline S.NO. & $\begin{array}{l}\text { Health resources } \\
\text { District Immunization Officer(Trained in last 3 years } \\
\text { for RI/CCVLM) }\end{array}$ & 3 & $3(100 \%)$ & 0 \\
\hline $\mathbf{1}$ & $\begin{array}{l}\text { Cold Chain Handlers(Trained in last 3 years for CCH } \\
\text { module) }\end{array}$ & 3 & $3(100 \%)$ & 0 \\
\hline $\begin{array}{l}\text { Cold Chain Technician(Trained in last 3 years for } \\
\text { CCE repair) }\end{array}$ & 3 & $2(66.7 \%)$ & $1(33.3 \%)$ \\
\hline $\mathbf{4}$ & ANM(Training on IPC \& mobilization for MI) & 1195 & $978(81.8 \%)$ & $217(18.2 \%)$ \\
\hline $\mathbf{5}$ & ASHA(Module 6\&7) & 3494 & $3168(91.7 \%)$ & $326(9.3 \%)$ \\
\hline $\mathbf{6 ( a )}$ & ASHA(Training on IPC \& mobilization for MI) & 3494 & $2948(84.3 \%)$ & $546(15.7 \%)$ \\
\hline $\mathbf{6 ( b )}$ & Th & Total & Trained & Not Trained \\
\hline At Planning Unit level & Health resources & 12 & $12(100 \%)$ & 0 \\
\hline $\mathbf{1}$ & Cold chain handlers(Training on MI) & 392 & $307(78.3 \%)$ & $85(21.7 \%)$ \\
\hline $\mathbf{2}$ & ANM(Training on IPC \& mobilization for MI) & 1452 & $1281(88.2 \%)$ & $171(11.8 \%)$ \\
\hline $\mathbf{3 ( a )}$ & ASHA(Module 6\&7) & 1452 & $946(65.2 \%)$ & $506(34.8 \%)$ \\
\hline $\mathbf{3 ( b )}$ & ASHA(Training on IPC \& mobilization for MI) & &
\end{tabular}

Above table depicts that most of the health care providers involved in mission indradhanush program were trained for their work at both district as well as planning unit level. Only percentages of ASHA were low for training of IPC \& mobilization as compared to training on module $6 \& 7$ at planning unit level.

Table No.3: Table showing information about session sites planned for Mission Indradhanush

\begin{tabular}{|l|l|c|}
\hline S.NO. & About session sites & Numbers \\
\hline $\mathbf{1}$ & Total number of sessions planned & 1368 \\
\hline $\mathbf{2}$ & No. of sessions receiving vaccine through AVD & $1182(86.4 \%)$ \\
\hline $\mathbf{3}$ & No. of sessions not receiving vaccine through AVD & $186(13.6 \%)$ \\
\hline $\mathbf{4}$ & No. of sessions taking $>1 \mathrm{hr}$ for receiving vaccine & $14($ about $1 \%)$ \\
\hline
\end{tabular}

Above table depicts about the total sessions planned for vaccination of children and pregnant women in three selected districts. About at $86 \%$ sessions sites vaccines was delivered by alternate vaccine delivery system while about $14 \%$ session sites was not covered by this system.

Table No.4: Table showing IEC activities performed at the level of District hospital and Planning units $(\mathrm{N}=15)$ for Mission Indradhanush

\begin{tabular}{|l|l|c|}
\hline S.No. & \multicolumn{1}{|c|}{ IEC material used } & Numbers \\
\hline $\mathbf{1}$ & Posters & 8533 \\
\hline $\mathbf{2}$ & Banners & 3832 \\
\hline $\mathbf{3}$ & AV Shows & 43 \\
\hline $\mathbf{4}$ & School rallies & 65 \\
\hline $\mathbf{5}$ & Mother meetings & 1671 \\
\hline $\mathbf{6}$ & Miking / Drumings & 312 \\
\hline
\end{tabular}

Above table depicts about various IEC activities performed by three selected districts for increasing awareness and to increase vaccination coverage by covering those children who were either drop out or left out. 
Table No.5: Information about health functionaries, IEC material and vaccination material presents at the session sites $(\mathrm{N}=24)$ on the day of assessment

\begin{tabular}{|l|l|c|c|}
\hline S.No. & Health functionaries/IEC/Vaccination material & Presents & Absents \\
\hline $\mathbf{1}$ & ANM & $24(100 \%)$ & 0 \\
\hline $\mathbf{2}$ & ASHA & $19(79.2 \%)$ & $5(20.8 \%)$ \\
\hline $\mathbf{3}$ & AWW & $13(54.2 \%)$ & $11(45.8 \%)$ \\
\hline $\mathbf{4}$ & Supervisor health/MI(LHV/MO) & $5(20.8 \%)$ & $19(79.2 \%)$ \\
\hline $\mathbf{5}$ & Posters & $15(62.5 \%)$ & $9(37.5 \%)$ \\
$\mathbf{6}$ & Banners & $6(25 \%)$ & $18(75 \%)$ \\
\hline $\mathbf{7}$ & Pamphlets & $24(100 \%)$ & 0 \\
\hline $\mathbf{8}$ & MCP / immunization card & $19(79.2 \%)$ & $5(20.8 \%)$ \\
\hline $\mathbf{9}$ & Updated due list & $19(79.2 \%)$ & $5(20.8 \%)$ \\
\hline $\mathbf{1 0}$ & Hub cutter for needle cutting & $5(20.8 \%)$ & $19(79.2 \%)$ \\
\hline
\end{tabular}

Above table depicts that ANM (100\%) and ASHA workers $(80 \%)$ were presents and AWWs was not presents on most of the session sites on the day of assessment. Pamphlets and posters related to the mission indradhanush was available at most of the session sites while availability of banners was found low. Immunization card and updated due list by vaccinators was available at most of the session sites while availability of hub cutter for needle cutting was found low $(21 \%)$ at most of the session sites.

Table No.6: Assessment of Cold chain infrastructure and availability of logistics in cold chain room $(\mathrm{N}=15)$

\begin{tabular}{|c|c|c|c|}
\hline S.No. & Logistics & Yes & NO \\
\hline 1 & Separate stabilizer for CCE & $7(46.7 \%)$ & $8(53.3 \%)$ \\
\hline 2 & Wooden/Plastic blocks/Stand for CCE & $13(86.7 \%)$ & $2(13.3 \%)$ \\
\hline 3 & Dedicated room for cold chain, syringe $\&$ diluents & $11(73.3 \%)$ & $4(26.7 \%)$ \\
\hline 4 & Separate functional thermometer inside each CCE & $12(80 \%)$ & $3(20 \%)$ \\
\hline 5 & Dedicated table for icepacks conditioning & $7(46.7 \%)$ & $8(53.3 \%)$ \\
\hline 6 & Clean cloth for wiping icepacks after conditioning & $15(100 \%)$ & 0 \\
\hline 7 & $\begin{array}{l}\text { Separate temperature recording log book for each } \\
\text { CCE }\end{array}$ & $15(100 \%)$ & 0 \\
\hline 8 & Emergency plan visible & $14(93.3 \%)$ & $1(6.7 \%)$ \\
\hline 9 & Cabinet temperature for $\operatorname{ILR}\left(+2\right.$ to $\left.+8^{0} \mathrm{C}\right)$ & $15(100 \%)$ & 0 \\
\hline 10 & Cabinet temperature for $\mathrm{DF}\left(-15\right.$ to $\left.-25^{0} \mathrm{C}\right)$ & $15(100 \%)$ & 0 \\
\hline 11 & $\begin{array}{l}\text { Arrangement of vaccines according to their } \\
\text { sensitivity in ILR }\end{array}$ & $8(53.3 \%)$ & $7(46.7 \%)$ \\
\hline 12 & Proper arrangement of ice packs in DF & $11(73.3 \%)$ & $4(26.7 \%)$ \\
\hline
\end{tabular}

Above table depicts the infrastructure and logistics availability at cold chain points on the day of assessment. Most of the cold chain points had separate stand for CCE (87\%), room for placing vaccines, syringes and diluents (73\%), separate functional thermometer inside each $\operatorname{CCE}(80 \%)$, clean cloth for wiping icepacks, Separate temperature recording log book and visible emergency plan (93\%). Recommended temperature was maintained in ILR and DF both in all cold chain points while proper arrangements of icepacks in DF at about $73 \%$ cold chain points. Availability of separate stabilizer for CCE and dedicated table for icepacks conditioning was found relatively low. Arrangement of vaccines according to their sensitivity in ILR was also found only at $53 \%$ cold chain points.

\section{Results \& Discussion}

In this study it was found that most of the staff required in Mission Indradhanush program was available in all three districts. One post of cold chain technician was vacant in one district. During the interview of DIO, he stated that for the program they hired a technician temporarily. But training for repair of cold chain equipments in last 3 years was not attended by the technician required as per the guidelines.

In the present study all District Immunization Officers at district level \& Cold chain handlers at 
district and planning unit level were trained for the program. While in study done by Lalitha Krishnappa et al (2014) ${ }^{4}$ in which $85 \%$ cold chain handlers had trained on routine immunization in last two years. Study done by Dr. Dhrubajyoti Choudhury et al (2016) ${ }^{\mathbf{5}}$ in which $58.33 \%$ respondents trained in cold chain management.

In the present study at district level $82 \%$ ANMs and $84 \%$ ASHAs were trained about Interpersonnel communication and for mobilization for Mission Indradhanush and about 91\% ASHAs had trained about module $6 \& 7$. While at the level of planning unit training status of ANMs was found $78 \%$ and of ASHAs was found $65 \%$ about Interpersonnel communication and for mobilization for Mission Indradhanush. Similar finding were seen by Victoria Bolanle Brown et al $^{\mathbf{6}}$ that about $85.5 \%$ of the participants had previous immunization training while $14.5 \%$ never had any form of training on immunization. In a study done by Onprasonk Widsanugorn et al (2011) ${ }^{\mathbf{7}}$ $80 \%$ health workers had trained about EPI and cold chain system.

In the present study About $86 \%$ sessions sites was covered by alternate vaccine delivery system (AVD) for vaccine delivery to ANM while about $14 \%$ session sites was not covered by this system. While Deepak Saxena et al (2010) ${ }^{8}$ in their study stated that number of vaccination sessions increased by $62.30 \%$ compared to last year. In 146 villages mobility support of AVD was given to ANMs.

In the present study various IEC activities like posters, banners, AV shows, school rallies mother meetings etc was performed by three selected districts for increasing awareness and to increase vaccination coverage by covering those children who were either drop out or left out for vaccination. In study done by Asha Ram Tyagi et al (2015) ${ }^{9}$ found that IEC about immunization schedule was available in rural $78 \%$ and in urban $92 \%$.

In the present study separate stabilizers for each cold chain equipments was available only at $47 \%$ cold chain points while in study of Kedar $\mathbf{G}$ Mehta et al (2016) ${ }^{\mathbf{1 0}}$ where ILR and DF were connected to separate power stabilizers at $77.8 \%$ centres and in study of Dr. Dhrubajyoti Choudhury et $\mathbf{a l}^{\mathbf{5}}$ where ILR and DF were connected to functional Voltage Stabilizer in $75 \%$ cold chain points.

In present study wooden/plastic blocks/stand for CCE was available in $87 \%$ cold chain points. Separate functional thermometer inside each CCE was available in $80 \%$ cold chain points while in the study of Dr.Dhrubajyoti Choudhury et al ${ }^{\mathbf{5}}$ where functional thermometer was placed inside every ILR and DF of all the centres and in study done by Lalitha Krishnappa et al ${ }^{4}$ where functional thermometer was available in $91 \%$ centres. In another study done by Kedar G Mehta et $\mathbf{a l}{ }^{\mathbf{1 0}}$ working thermometer was found inside ILR at 18 centres and inside DF at all 19 centres. Santosh M Biradar et al (2013) ${ }^{\mathbf{1 1}}$ found that in only $76.1 \%$ health centres ILR and DF were properly placed, ILR and DF were connected to functional Voltage Stabilizer in $91.3 \%$ health centres. A functional thermometer was placed inside ILR and DF only in $76.1 \%$ health centres. In present study dedicated table for icepacks conditioning was available at only $47 \%$ cold chain points. Separate temperature recording log book for each CCE was available at all cold chain points. Emergency plan was available at about 93\% cold chain points in where in study done by S.Mallik et al (2013) ${ }^{\mathbf{1 2}}$ they found unsatisfactory emergency contingency plan.

In present study posters and pamphlets of MI was available at most of session sites but availability of banners related to MI was found low (25\%). MCP / immunization card was available at most of session sites (79\%). Availability of Counter foil of the MCP / immunization card of previous session was found only at $50 \%$ session sites. Hub cutter was available only at the $20.8 \%$ session where in study of Santosh M Biradar et $\mathbf{a l}^{\mathbf{1 1}}$ functional hub cutter was available at $63.1 \%$ session sites. They also found that Due list of beneficiaries was maintained by $65.2 \%$ session sites where another 
study done by Tushar Patel et al (2011) ${ }^{13}$ observed that $54.5 \%$ Auxiliary Nurse Midwife (ANM) had maintained Due list. Shaheen Akhtar Choudhury et al (2016) ${ }^{\mathbf{1 4}}$ found that among the 23 ANM only $43.5 \%$ had available action plan at the session site. Updated beneficiary list was available was available with $78.3 \%$ of ANM.

In present study training on IPC \& mobilization for MI was incomplete in some ANMs and ASHA workers as required for the program. On asking it was found that most of the trainees were either posted for some governmental activities or went to Ujjain for Simhasth (Kumbh Mela) at training period. Banners were not available at many session sites because not provided from the district due to insufficient funds for IEC. Icepacks were not arranged in criss-cross manner in DF in some cold chain points. On asking to cold chain handlers they told that due to Pulse Polio program there was requirements of extra icepacks and hence they kept large numbers of icepacks in DF haphazardly.

\section{Conclusion}

The findings of this study revealed that most of the health workers (DIO, MO, CCH, ANM and ASHA) involved in Mission Indradhanush were trained. Basic infrastructure and logistics required for cold chain maintenance was available at most of the cold chain points. However separate stabilizer for each CCE and dedicated table for icepacks preparation were unavailable at most of the cold chain points. Material for vaccination like MCP cards, counter foils and updated due list were available at most of the session sites. However hub cutters for needle cutting were not available at most of the session sites.

\section{Recommendation}

1) The vacant post of cold chain technician should be filled as earliest for proper functioning of the programme.

2) Although regular training of health workers is done annually retraining/ refresher sessions should be arranged at regular interval.

3) Logistics such as power stabilizer, dedicated table for ice-packs preparation and hub cutter needs to be procured for successful running of immunization programme.

Funding: No funding sources

Conflict of interest: None declared

Ethical approval: This study was approved by the institutional ethics committee.

\section{References}

1. Vashistha PM, Kumar P. 50 years of Immunization in India: Progress and Future; Indian Pediatrics. 2013; 50:111-18

2. Keshavamurthy H.R. Director in Press Information Bureau, Kolkata. Mission Indradhanush to Put Vaccination Efforts on High Speed;2015

3. Mission Indradhanush Operational guidelines 2015

4. Krishnappa L, Anniappan AB, Voderhobli N.H Evaluation of cold chain practices in urban health centers of a metro city in India. National Journal of Community Medicine 2014;5:288-92

5. Choudhury D, Baruah R.Knowledge and practices of cold chain handler regarding cold chain equipment and vaccines storage in Chirang district, Assam, International Journal of Current Research 2016;8:35464-67

6. Brown VB, Oluwatosin OA.Impact of training intervention on immunization providers' knowledge and practice of routine immunization in Ibadan, southwestern Nigeria .The Pan African Medical Journal. 2017;26:216

7. Widsanugorn O. Healthcare workers' knowledge and practices regarding expanded program on immunization in Kalasin, Thailand j med sci. 2011 ;73: 177-185 
8. Saxena D and Ganguly P S. Evaluation of new alternate vaccine delivery in Shivpuri District of Madhya Pradesh

9. Tyagi AR, Pradhan S. Assessment of Beneficiary Response about Immunization and Health Services Provided at Anganwadi Centres of Jabalpur District. N J of Community Med 2015; 6:536-40.

10. Mehta KG, Pandya CM, Chavda PD, Solanki DM. Study of cold chain management system at Urban Primary Health Centers (UPHCs) of Vadodara city in Western India. Int $\mathbf{J}$ Med Sci Public Health (Online First) 2015;4:1-4

11. Biradar SM . Evaluation of Vaccine Storage Practices in Primary Health Centres of Bijapur District of Karnataka. International Journal of Pharma and Bio Sciences, 2013; 4 : 1290- 93.

12. Mallik S, Mandal PK. Assessing cold chain status in a metro city of India: an intervention study. Afr Health Sci. 2011;11:128-33.

13. Patel TA, Pandit NB . Why infants miss vaccination during routine immunization sessions? Study in a rural area of Anand district, Gujrat IJPH .2011;55, Issue:32123

14. Choudhury SA, Ojah J. A study on knowledge and practice of immunization services among auxiliary nurse midwives of subcentre at Chirang district, assam J. Evid. Based Med. Healthc. 2016; 3: 618621. 